\title{
NOTE ON THE CONTINUITY OF THE ERGODIC FUNCTION
}

\author{
BY M. H. MARTIN
}

1. Introduction. Let $M$ denote a bounded point set and $\epsilon$ an arbitrarily chosen positive number. A continuous curve $C$ is termed $\epsilon$-ergodic to $M$ if an arbitrary point of $M$ lies at a distance $\leqq \epsilon$ from some point of $C$. Recently* I have shown that the set of continuous, rectifiable curves $\epsilon$-ergodic to $M$ contains a member whose length furnishes an absolute minimum for the lengths of the curves in the set. This member was called an ergodic curve of $M$ and its length the ergodic function $\Lambda(\epsilon)$ of $M$. The function $\Lambda(\epsilon)$ is finite and non-negative, being equal to zero if and only if $\epsilon \geqq \rho$, where $\rho$ is the radius of the smallest circular region containing $M$. In addition $\Lambda(\epsilon)$ was proved to be a monotone non-increasing function of $\epsilon$ which is always continuous on the right.

In this note it is shown that $\Lambda(\epsilon)$ is also continuous on the left (and is therefore continuous in the ordinary sense). In the original version of this paper I was able to prove this result only for a value $\epsilon_{0}(<\rho)$ of $\epsilon$ for which the set $M$ had an ergodic curve which was an "ordinary curve" (a continuous curve which is either of class $C^{\prime}$ or else made up of a finite number of arcs of class $C^{\prime}$ ). The general result announced above is made possible by Lemma 2 below for which I am indebted to Professor von Neumann.

2. Preliminary Lemmas. In this section we shall assemble a number of lemmas leading to the proof of the result announced in the introduction.

Lemma 1. The set $M_{1}$ of points lying at a distance $\leqq$ from the points of a continuous rectifiable arc of length $2 s$ joining two points $A$ and $B$ situated $a$ distance $2 c(c \leqq s)$ apart lies in a region composed of the points interior to two circles described about $A$ and $B$ as centers with radii equal to $\epsilon+\left(2^{1 / 2} \alpha+s /(2 \epsilon)\right) s$, where $\alpha^{2}=1-c / s$. 734.

* Ergodic curves, American Journal of Mathematics, vol. 58 (1936), pp. 727- 
For the proof of this lemma we first observe that a point on the rectifiable arc either lies in the interior of an ellipse $E$ having $A$ and $B$ as foci and a major axis of length $2 s$, or lies on $E$ itself. The proof of this fact is elementary and is omitted.

The set $E_{\epsilon}$ of points lying at a distance $\leqq \epsilon$ from either the points of $E$ or the points interior to $E$ therefore contains the set $M_{1}$.

The boundary of $E_{\epsilon}$ is a closed analytic curve forming part of the envelope of the family of circles of radius $\epsilon$ with centers on $E$.

$E_{\epsilon}$ is divided into two halves by the minor axis of $E$ produced. Let us consider that half which contains the focus $A$. An elementary calculation shows that an absolute maximum $R$ of the distances of the points of this half of $E_{\epsilon}$ from $A$ is furnished by the two points in which the minor axis of $E$ produced intersects the boundary of $E_{\epsilon}$. Upon computing this distance, we find

$$
R=\left[\epsilon^{2}+2 \epsilon\left(s^{2}-c^{2}\right)^{1 / 2}+s^{2}\right]^{1 / 2} .
$$

Since $c \leqq s$ and $s-c=\alpha^{2} s$, it follows that

$$
R \leqq\left[\epsilon^{2}+2^{3 / 2} \epsilon \alpha s+s^{2}\right]^{1 / 2}<\epsilon+\left(2^{1 / 2} \alpha+\frac{s}{2 \epsilon}\right) s .
$$

A circle of radius $\epsilon+\left(2^{1 / 2} \alpha+s /(2 \epsilon)\right) s$ described about $A$ as a center accordingly contains in its interior the half of $E_{\epsilon}$ containing $A$. A similar circle described about $B$ as a center will contain the remaining half of $E_{\epsilon}$ in its interior. The two circles taken together will then contain the whole of $E_{\epsilon}$ and will therefore $a$ fortiori contain the set $M_{1}$ in their interiors.

LEMma 2. Let

$$
C: x=x(s), \quad y=y(s), \quad 0 \leqq s \leqq L, \quad(s=\text { arc length }),
$$

be an open continuous rectifiable curve of length $L$. Let $C$ be divided into $n$ arcs of lengths $s_{1}^{(n)}, s_{2}^{(n)}, \cdots, s_{n}^{(n)}$ subtending chords of lengths $c_{1}^{(n)}, c_{2}{ }^{(n)}, \cdots, c_{n}^{(n)}$ respectively. Define the $n$ quantities $\alpha_{1}^{(n)}, \alpha_{2}^{(n)}, \cdots, \alpha_{n}^{(n)}$ by the equations

$$
\alpha_{i}^{(n)}=\left[1-\frac{c_{i}^{(n)}}{s_{i}^{(n)}}\right]^{1 / 2}, \quad(i=1,2, \cdots, n),
$$

and then the $n+1$ quantities $\bar{\alpha}_{0}^{(n)}, \bar{\alpha}_{1}^{(n)}, \cdots, \bar{\alpha}_{n}^{(n)}$ by the relations 


$$
\begin{aligned}
& \bar{\alpha}_{0}^{(n)}=\alpha_{1}^{(n)}, \quad \bar{\alpha}_{i}^{(n)}=\alpha_{i}^{(n)}+\alpha_{i+1}^{(n)} \quad \text { for } \quad i=1,2, \cdots, n-1, \\
& \bar{\alpha}_{n}^{(n)}=\alpha_{n}^{(n)} .
\end{aligned}
$$

If $s_{1}^{(n)}=s_{2}^{(n)}=\cdots=s_{n}^{(n)}=L / n$, the arithmetic mean

$$
\bar{\alpha}_{n}=\frac{1}{n+1} \sum_{i=0}^{n} \bar{\alpha}_{i}^{(n)}
$$

of $\bar{\alpha}_{0}^{(n)}, \bar{\alpha}_{1}^{(n)}, \cdots, \bar{\alpha}_{n}^{(n)}$ tends to zero as $n$ tends to infinity.

We have

$$
\bar{\alpha}_{n}=\frac{2}{n+1} \sum_{i=1}^{n} \alpha_{i}^{(n)}<\frac{2}{n} \sum_{i=1}^{n} \alpha_{i}^{(n)},
$$

and therefore, upon substituting the values of $\alpha_{i}^{(n)}$ given above and applying Schwarz's inequality.

$$
\bar{\alpha}_{n}<\frac{2}{n}\left[n \sum_{i=1}^{n}\left(1-\frac{c_{i}^{(n)}}{s_{i}^{(n)}}\right)\right]^{1 / 2},
$$

whereupon, since $s_{i}^{(n)}=L / n$, it follows that

$$
\bar{\alpha}_{n}<2\left[1-\frac{\sum_{i=1}^{n} c_{i}^{(n)}}{L}\right]^{1 / 2} .
$$

Now $C$ is rectifiable of length $L$. Therefore $\lim _{n \rightarrow \infty} \sum_{i=1}^{n} c_{i}^{(n)}=L$, and consequently $\lim _{n \rightarrow \infty} \overline{\bar{\alpha}}_{n}=0$.

LEMMA 3. Let

$$
C: x=x(s), \quad y=y(s), \quad 0 \leqq s \leqq L,
$$

be a continuous rectifiable curve of length $L$. Denote by $C_{\epsilon}$ the set of points lying at a distance $\leqq$ from the points of $C$. There exists a sequence $\left\{C_{n}\right\}$ of continuous rectifiable curves for which, if $L_{n}$ denotes the length of $C_{n}$, the following hold:

(a) $\lim L_{n}=L$,

(b) $C_{n}$ is $\epsilon_{n}$-ergodic to $C_{\epsilon}$ with $\epsilon_{n}<\epsilon$ and $\lim \epsilon_{n}=\epsilon$.

In proving this lemma it is convenient to take $C$ to be an open curve, the proof permitting an immediate adaptation to the case where $C$ is closed. Divide $C$ up into $n$ equal arcs each 
of length $L / n$ by inserting $n+1$ points on $C$. These $n+1$ points we shall denote by $0,1,2, \cdots, n$, where 0 and $n$ denote the endpoints of $C$.

About a point $i,(i=0,1,2, \cdots, n)$, of the subdivision we describe two circles, one, $\Gamma_{i}$, of radius $\epsilon+\left(2^{1 / 2} \bar{\alpha}_{i}{ }^{(n)}+L /(4 n \epsilon)\right) L /(2 n)$, the other, $\gamma_{i}$, of radius $\left(2^{1 / 2} \bar{\alpha}_{i}^{(n)}+L /(4 n \epsilon)\right) L / n$, where in each case $\bar{\alpha}_{i}^{(n)}$ denotes the quantity defined in Lemma 2 .

The totality of points interior to the circles $\Gamma_{i}$ constitutes a point set which we designate by $M_{n}$. On placing $s=L /(2 n)$ in Lemma 1, we ascertain that $C_{\epsilon} \subset M_{n}$.

A point making a complete circuit of a circle $\gamma_{i}$ comes within a distance $\leqq\left(2^{1 / 2} \bar{\alpha}_{i}^{(n)}+L /(4 n \epsilon)\right) L / n$ of the points enclosed by $\gamma_{i}$, and within a distance $\leqq \epsilon-\left(2^{1 / 2} \bar{\alpha}_{i}{ }^{(n)}+L /(4 n \epsilon)\right) L /(2 n)$ of those points outside $\gamma_{i}$ but inside $\Gamma_{i}$. For sufficiently great values of $n$ the latter of the two distances is the greater. Consequently, for sufficiently great values of $n$, a point making a complete circuit of $\gamma_{i}$ comes within a distance $\leqq \epsilon-\left(2^{1 / 2} \bar{\alpha}_{i}^{(n)}+L /(4 n \epsilon)\right) L /(2 n) \leqq \epsilon-L^{2} /\left(8 n^{2} \epsilon\right)$ of every point interior to $\Gamma_{i}$.

Now consider a point $P$ which traverses $C$ from one endpoint to the other and which makes complete circuits of all the circles $\gamma_{i}$ during the process. More precisely, $P$ moves as follows: starting at the endpoint 0 of $C$, the point $P$ moves along $C$ in the direction of increasing arc length until it meets the circle $\gamma_{0}$ with center 0 for the first time; $P$ then makes a complete circuit of $\gamma_{0}$; after returning to the curve $C$, the point $P$ continues along $C$ in the direction of increasing arc length until it meets the circle $\gamma_{1}$ with center 1 for the first time; $P$ then makes a complete circuit of $\gamma_{1} ; \cdots$. At the completion of this process $P$ coincides with $n$ and will have traced out a continuous rectifiable curve $C_{n}$ of length $L_{n}$, where

$$
L_{n}=L+\sum_{i=0}^{n} 2 \pi\left(2^{1 / 2} \bar{\alpha}_{i}^{(n)}+\frac{L}{4 n \epsilon}\right) \frac{L}{n} .
$$

On replacing $\sum_{i=0}^{n} \bar{\alpha}_{i}{ }^{(n)}$ by its value $(n+1) \overline{\bar{\alpha}}_{n}$ (introduced in Lemma 2) and carrying out the remainder of the summation, we find

$$
L_{n}=L\left[1+2 \pi \frac{n+1}{n}\left(2^{1 / 2} \overline{\bar{\alpha}}_{n}+\frac{L}{4 n \epsilon}\right)\right] .
$$


In addition the curve $C_{n}$ is $\epsilon_{n}$-ergodic to $M_{n}$, where

$$
\epsilon_{n}=\epsilon-\frac{L^{2}}{8 n^{2} \epsilon}<\epsilon .
$$

The proof of the lemma is now completed by observing that, since $C_{\epsilon} \subset M_{n}$, the curve $C_{n}$ is $\epsilon_{n}$-ergodic to $C_{\epsilon}$, and that

$$
\lim \epsilon_{n}=\epsilon, \quad \lim L_{n}=L,
$$

the latter of which follows from Lemma 2.

3. The Continuity of $\Lambda(\epsilon)$. Let $\epsilon_{0}$ denote a fixed value of $\epsilon$ lying in the interval $0<\epsilon<\rho$ (for the definition of $\rho$ see the introduction). Let $C$ be an ergodic curve of $M$ for $\epsilon=\epsilon_{0}$. Write $\Lambda\left(\epsilon_{0}\right)=L$, so that the length of $C$ is $L$. Let $C_{\epsilon_{0}}$ denote the set of points lying at a distance $\leqq \epsilon_{0}$ from points of $C$. We note that $M \subset C_{\epsilon_{0}}$. The ergodic function $\Lambda(\epsilon)$ will now be demonstrated to be continuous on the left for $\epsilon=\epsilon_{0}$.

If $\Lambda(\epsilon)$ be discontinuous on the left for $\epsilon=\epsilon_{0}$, we shall have, since $\Lambda(\epsilon)$ is monotone non-increasing

$$
\lim \Lambda\left(\epsilon_{n}\right)=L+\delta, \quad(\delta>0),
$$

for an arbitrarily selected sequence $\left\{\epsilon_{n}\right\}$ of $\epsilon$ values for which

$$
\epsilon_{n}<\epsilon_{0}, \quad \lim \epsilon_{n}=\epsilon_{0} .
$$

On the other hand, according to Lemma 3 there exists a sequence $\left\{C_{n}\right\}$ of continuous rectifiable curves such that, if $L_{n}$ denotes the length of $C_{n}$, we have

(a) $\lim L_{n}=L$,

(b) $C_{n}$ is $\epsilon_{n}$-ergodic to $C_{\epsilon_{0}}$ (and therefore $\epsilon_{n}$-ergodic to $M$ ) with $\epsilon_{n}<\epsilon_{0}$ and $\lim \epsilon_{n}=\epsilon_{0}$.

Therefore, since $\Lambda\left(\epsilon_{n}\right) \leqq L_{n}$, it follows that

$$
\lim \Lambda\left(\epsilon_{n}\right) \leqq L,
$$

which is a contradiction to our previous result. Hence $\Lambda(\epsilon)$ is continuous on the left for $\epsilon=\epsilon_{0}$.

The case $\epsilon_{0}<\rho$ is now disposed of. There remains the case $\epsilon_{0}=\rho$ to consider. That $\Lambda(\epsilon)$ is continuous on the left in this case is trivial. The ergodic curve $C$ shrinks to a point $P$, since $\Lambda(\rho)=0$, and one replaces the curve $L_{n}$ above by a circle of radius $1 / n$ described about $P$ as center.

UNIVERSITY OF MARYLAND 